\title{
Mechanistic Study of Palladium-Catalyzed Wacker-Type Cyclizations
}

Metal-Catalyzed Asymmetric

Synthesis and

Stereoselective

Reactions

\section{Key words}

Wacker-type cyclization

palladium

pyrrolidines
Significance: Recently, Stahl and co-workers had shown that a $\mathrm{Pd}(\mathrm{II})$ catalyst with a chiral pyridine-oxazoline (pyrox) ligand allowed preparation of pyrrolidines in high yield and enantioselectivity (Org. Lett. 2011, 13, 2830). In the enantioselective cyclization of $\gamma$-alkenyl tosylamides, the anionic ligand (TFA vs OAc) was found to have a significant impact on the reaction outcome, where the use of $\left[\mathrm{Pd}(\right.$ pyrox $\left.)(\mathrm{OAc})_{2}\right]$ gave significantly diminished yield and enantioselectivity. Through a series of mechanistic investigations with a chiral, deuterated substrate probe, the authors showed the significant effect the anionic ligand has in selecting the nucleopalladation (NP) pathway of the Wacker-type cyclization, which in course determines the ancillary neutral donor's ability to alter the stereochemical course of the pathway. This data provides the first direct correlation between NP stereoselectivity and the enantioselectivity of the transformation.
Comment: By using ${ }^{1} \mathrm{H}$ NMR spectroscopy and HPLC analyses to determine H/D ratios and enantiomeric excesses, the authors were able to determine the yields of the four possible products from the reaction of a deuterated acyclic substrate under different conditions (see above). They showed that only in the trans pathway does the pyrox ligand play a significant role, thus the trans-amidopalladation (AP) pathway proceeds with high enantioselectivity, while the cis-AP pathway exhibits low enantioselectivity. The authors suggest that the carboxylate ligand acts as a Brønsted base to mediate $\mathrm{Pd}$-amidate bond formation in the cis-AP pathway, whereas the TFA anionic ligand is substituted by the substrate alkene and favors the trans-AP pathway. 